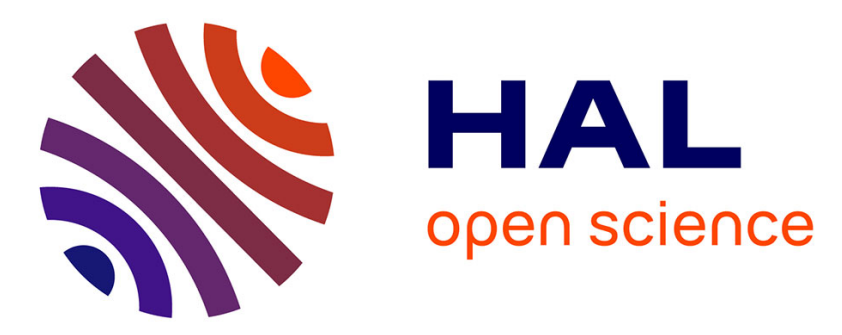

\title{
A MODEL FOR ULTRASONIC EFFECTS DURING AN FCC-HCP MARTENSITIC TRANSFORMATION
}

\author{
A. Granato, R. Schwarz, G. Kneezel
}

\section{To cite this version:}

A. Granato, R. Schwarz, G. Kneezel. A MODEL FOR ULTRASONIC EFFECTS DURING AN FCC-HCP MARTENSITIC TRANSFORMATION. Journal de Physique Colloques, 1981, 42 (C5), pp.C5-1055-C5-1059. 10.1051/jphyscol:19815163 . jpa-00221037

\section{HAL Id: jpa-00221037 https://hal.science/jpa-00221037}

Submitted on 1 Jan 1981

HAL is a multi-disciplinary open access archive for the deposit and dissemination of scientific research documents, whether they are published or not. The documents may come from teaching and research institutions in France or abroad, or from public or private research centers.
L'archive ouverte pluridisciplinaire HAL, est destinée au dépôt et à la diffusion de documents scientifiques de niveau recherche, publiés ou non, émanant des établissements d'enseignement et de recherche français ou étrangers, des laboratoires publics ou privés. 


\title{
A MODEL FOR ULTRASONIC EFFECTS DURING AN FCC-HCP MARTENSITIC TRANSFORMATION *
}

\author{
A.V. Granato, R.B. Schwarz ${ }^{*}$ and G.A. Kneeze1 ${ }^{* *}$ \\ Physics Department and Materials Research Laboratory, University of IlZinois \\ at Urbana-Champaign, Urbana, IL 61801, U.S.A.
}

\begin{abstract}
It is proposed that the FCC-HCP martensitic phase transformation may be studied ultrasonically by measuring the effect of "optical" vibrations of extended dislocations on the [111] shear sound velocity and attenuation. A simple model for the behavior of such dislocations predicts a dip in velocity and $a$ rise in attenuation as the transformation is approached.
\end{abstract}

It is generally believed that dislocations play a role in martensitic transformations, but the details of the role are obscure. A simple model for the FCC-HCP case is developed here which should be susceptible to experimental checks by ultrasonic measurements.

Dislocations in fcc metals are extended ${ }^{1}$ with a width that depends on the stacking fault energy. In a material which transforms from fcc to hcp, these extended dislocations are likely nucleation centers as first proposed by Christian. 2 When the free energy difference between the phases decreases, the extended dislocations (each boundary a two-layer hep fault) widens. Ericson ${ }^{3}$ has observed such widening via electron microscopy in cobalt and cobalt-nickel. His measurements were not made close to the transformation temperature $M_{s}$. He suggested that the stacking fault energy of an isolated extended dislocation is linear in temperature but does not go to zero at $\mathrm{M}_{\mathrm{s}}$. Olson and cohen $^{4}$ proposed subsequently that the nucleatiing defects are overlapping extended dislocations spaced every other layer to produce a thicker hcp fault with lower total energy than an equal number of two layer faults. In both schemes 2,4 it is suggested that extended dislocations gradually widen as the transformation is approached and then propagate across the plane as the free energy difference becomes negative.

Because the partial dislocations bounding the fault have anti-parallel components, it may be possible (using a suitably oriented stress wave) to excite an "optical mode" of vibration in which the partials move in opposite directions. As the binding force between the partials decreases, the resonant frequency of the optical mode decreases. Ultrasonic measurements of the velocity and attenuation of

\footnotetext{
* Work supported by the National Science Foundation, under grant NSF DMR77-10556

*Present address : Materials Science Dept., Argonne National Lab., Argonne, IL 60439, U.S.A.

** Present address : Xerox Research Laboratory, Webster, NY 14580, U.S.A.
} 
stress waves exciting the optical mode may therefore be a means of detecting the dislocation widening as the transformation temperature is approached.

A rectangular loop model can be used to find the behavior of the equilibrium width and optical mode resonant frequency (Flgure 1). The extended dislocation has a length $\mathrm{L}$ and width $\mathrm{w}$. The energy terms depending on $\mathrm{w}$ are $\mathrm{E}_{\mathrm{R}}$ (the repulsive interaction of the partials), $\mathrm{E}_{\mathrm{T}}$ (The line energy of the vertical segments which lengthen as $w$ increases), $E_{\sigma}$ (the strain energy interaction with external stress), and $E_{Y}$ (the stacking fault energy). Point defect pinning is neglected, but this model does incorporate $\mathrm{E}_{\mathrm{T}}$ and $\mathrm{E}_{\sigma}$, which simpler treatments neglect. $6,7 /$.

The repulsive energy $E_{R}$ for an isotropic solid is obtained by integrating the radial component of the interaction force given by

$$
\frac{G b_{p}^{2}{ }_{L}}{2 \pi w}\left[\cos \theta_{1} \cos \theta_{2}+\frac{\sin \theta_{1} \sin \theta_{2}}{1-\nu}\right]^{6 /} \text {, where } \theta_{1}=\beta-30^{\circ}
$$

Fig. 1 - A. The rectangular loop model for an extended dislocation of length $L$ and width $w$. The Burgers vector of the complete dislocation $\vec{b}$ is assumed to be at an angle $\beta$ from the dislocation direction, while the angle between $\vec{b}$ and the shear stress on the plane is $\alpha$.

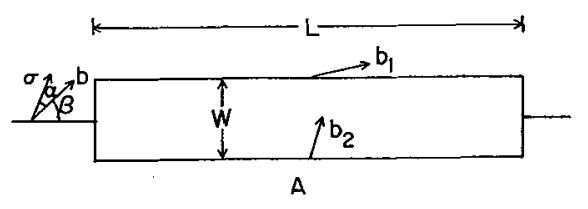

$$
\text { B. The four energy terms versus }
$$

width $w . E_{\sigma}$ and $E_{\gamma}$ may be positive or negative and are grouped together for these possibilities.

C. The total energy versus width $w$. For negative $E_{\gamma}+E_{\sigma}$ the equilibrium width is larger and the curvature at the minimum is smaller.
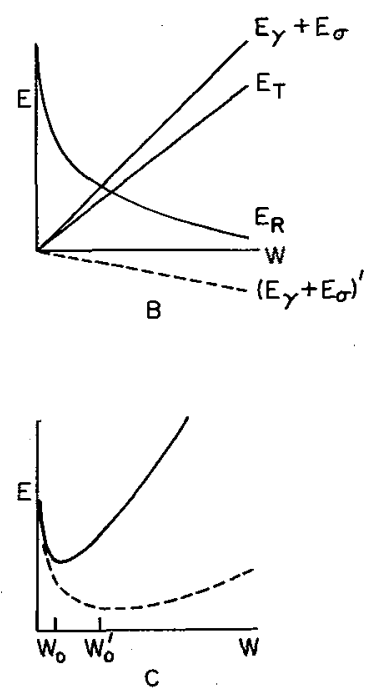

and $\theta_{2}=\beta+30^{\circ}$ are the angles between $\vec{b}_{1}, \vec{b}_{2}$ and the segments of the dislocation line horizontal in Fig. 1. The line energy $\mathrm{E}_{\mathrm{T}}$ is obtained by summing over the four segments vertical in Fig. 1 of

$$
E_{T}=\frac{G b_{p}^{2}}{4 \pi} \frac{w}{2} \ln \frac{L}{b}\left\{\sum_{i=1}^{4}\left(\cos ^{2} \theta_{1}+\frac{\sin ^{2} \theta_{i}}{1-v}\right)\right\} \text {. }
$$


with $\theta_{1}=\beta-120^{\circ}, \theta_{2}=\beta+60^{\circ}, \theta_{3}=\beta+120^{\circ}$, and $\theta_{4}=\beta-60^{\circ}$. The strain energy $E_{T}$ arising from the separation of the partials is found from the force $F_{1}$ on the partials horizontal in Fig. 1 given by $F_{i}=\sigma b_{p} L \cos \phi_{i}$, where $\phi_{i}$ is the angle between the component of stress on the slip plane and $\vec{b}_{1}$ or $\vec{b}_{2}, \phi_{1}=\alpha+30$ and $\phi_{2}=\alpha-30$. This yields $E_{\sigma}=-\sigma b_{p} L(w / 2)[\cos (\alpha+30)-\cos (\alpha-30)]$. The results are

$$
\begin{aligned}
& E_{R}=-\frac{G b_{p}^{2} L}{2 \pi} f_{1}(\beta) \ln w, \\
& E_{T}=\frac{G b_{p}^{2}}{4 \pi} f_{2}(\beta) w, \\
& E_{\sigma}=\sigma_{E} L b{ }_{p} f_{3}(\alpha) w, \text { and } \\
& E_{Y}=\gamma L w,
\end{aligned}
$$

where $b_{p}$ is the partial dislocation Burgers vector, $B$ is the angle between the Burgers vector $\vec{b}$ of the complete dislocation and its direction, $\sigma_{E}$ is the component of external stress on the (111) transformation and slip plane, $\alpha$ is the angle

between $\sigma_{E}$ and $\vec{b}$, and $\gamma$ is the stacking fault energy. In the above $f_{1}, f_{2}$ and $f_{3}$ are factors depending upon the orientation of the Burgers vectors, but are independent of $w, \sigma$, and $\gamma$ :

$$
\begin{aligned}
& f_{1}(\beta)=\frac{1}{4}\left[\frac{2-\nu-2 \nu \cos \beta}{1-\nu}\right] \\
& f_{2}(\beta)=\left\{\frac{4-\nu\left[1+2 \sin ^{2} \beta\right]}{2(1-\nu)}\right\} \ln \frac{L}{b} \\
& f_{3}(\alpha)=(\sin \alpha) / 2
\end{aligned}
$$

For $v=1 / 3$, as $\beta$ goes from 0 to $90^{\circ}, f_{1}$ varies between $3 / 8$ and $7 / 8$, while $\mathrm{f}_{2} / \ln (\mathrm{L} / \mathrm{b})$ varies between $11 / 4$ and $9 / 4$.

$E_{Y}$ and $E_{\sigma}$ may be positive or negative, depending on the temperature and the direction of the applied stress. They are therefore plotted together in Figure 1. $\mathrm{E}_{\mathrm{T}}$ is always positive, as it always takes energy to create the extra length of dislocation necessary to widen the fault. The creation of the extra length of dislocation is one source of the hysteresis observed in the transformation. The free energy difference between the two phases must not merely go to zero but must become sufficiently negative to minimize the total energy of the fault as it grows. The term $E_{\sigma}$ suggests that with properly applied external stress one may favor or hinder the widening of the fault, and in this way narrow or widen the hysteresis of the transformation. Weston and Granato 8 / have calculated for cobalt-nickel that the hysteresis could be narrowed by about $20^{\circ} \mathrm{C}$ at shear stresses of the order necessary to inftiate plastic deformation. 
The equilibrtum width $w_{0}$ is found by setting $(\mathrm{dE} / \mathrm{dw})=0$ :

$$
w_{0}=\frac{\frac{2 f_{1}(\beta)}{4 \pi\left(\gamma+\sigma_{E} b_{p} f_{3}(\alpha)\right)}+\frac{f_{2}(\beta)}{L}}{G b_{p}^{2}}
$$

For isolated extended dislocations the second term in the denominator (corresponding to the line energy barrier $\mathrm{E}_{\mathrm{T}}$ ) suggests that the transformation begins at the longest dislocation and is driven by a negative stacking fault or stress energy term. Since not all dislocations are the same length the transformation occurs over a range of temperatures rather than at a single temperature.

Based on the rectangular loop model we calculate the decrement and fractional change in velocity for an applied stress of amplitude $\sigma_{0}$ and frequency $\omega$. With no static applied stress, the equation of motion for the optical mode is

$$
A \ddot{y}+B \dot{y}+D y=b o_{0} \cos \omega t
$$

where $A$ is the effective dislocation mass, $B$ is the damping constant, and $D$ is a spring constant given by

$$
D=-\left.\frac{1}{L} \frac{d^{2} E}{d w^{2}}\right|_{w_{0}}=\frac{G b_{p}^{2} f_{1}(B)}{2 \pi w_{0}^{2}}
$$

The resulting change in shear modulus and the decrement are

$$
\begin{aligned}
& \frac{\Delta G}{G}=\frac{A v^{2}\left(\omega_{R}^{2}-\omega^{2}\right)}{\left[\left(\omega_{R}^{2}-\omega^{2}\right)^{2}+\left(\frac{\omega B}{A}\right)^{2}\right]}=-2 \frac{\Delta v}{v} \\
& \Delta=\frac{\pi \Lambda v^{2} \omega B}{\left[\left(\omega_{R}^{2}-\omega^{2}\right)^{2}+\left(\frac{\omega B}{A}\right)^{2}\right]}
\end{aligned}
$$

Fig. 2 - The fractional change in shear modulus $-(\Delta G / G)$ and the decrement $\Delta$ plotted versus the extended dislocation optical resonant frequency $\omega_{R_{5}}$ for $\Lambda=10^{4}$, $\mathrm{B}=10^{-4}, \omega=2 \pi \times 10^{7}, \mathrm{v}=3 \times 10^{5}$, and $\mathrm{A}=2 \times 10^{-15}$.

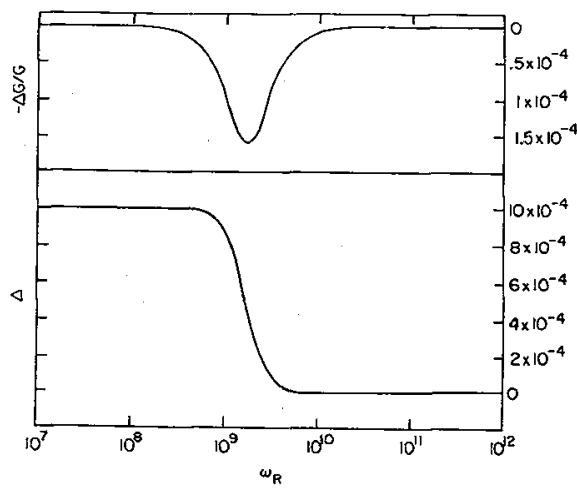


where $\omega_{R}=(D / A)^{1 / 2} \sim .3 v / w_{0}, v^{2}=G / \rho$, and $\Lambda$ is the density of extended dislocations having an optical mode resonant frequency $\omega_{R^{*}}$. In Figure $2, \frac{\Delta G}{G}$ and $\Delta$ are plotted versus $\omega_{R}$ assuming $\Lambda=10^{4} \mathrm{~cm}^{-2}$ and $B=10^{-4^{R}}$ in cgs units. Because $\gamma$ is approximately linear in temperature near the transformation, it is seen from Eqs. 5 and 7 that the $\omega_{R}$ axis may be viewed as a temperature axis. Far above the transformation temperature $\mathrm{M}_{\mathrm{S}}$, the equilibrium width of the fault is small and $\omega_{R}$ is much larger than the measurement frequency $\omega$. For $\omega_{R}$ large compared to the measurement frequency, the dislocation motion is in phase with the applied stress, but smal1, with $\Delta v / v \sim 1 / \omega_{R}{ }^{2}$. For $\omega_{R}$ small compared to the measurement frequency the dislocation motion is out of phase with the applied stress and small again, with $\Delta v / v \sim w_{R}^{2}$. In between, a maximum in the velocity change is obtained. The decrement meanwhile increases from its pretransformation value by an amount $\left(4 \pi \Delta v_{\max }\right) / v$.

The model thus predict a dip in velocity and a corresponding rise in decrement as $M_{s}$ is approached. These effects must be superposed on the background (a linear increase in velocity with decreasing temperature and a temperature independent decrement). Flnally as the transformation occurs, the quasi-static picture of the model is no longer applicable. Large numbers of dislocations are believed to move with velocities approaching the velocity of sound.5/ There will also be scattering due to the presence of two phases with different elastic constants leading to a dramatic increase in decrement and decrease in velocity.

In summary, the optical mode vibration model predicts a dip in velocity and a corresponding rise in decrement as the $M_{s}$ temperature is approached. What is more, these precursor effects can in principle be observed reversibly without transforming the sample.

\section{References}

1) R. D. Heidenreich and W. Shockley, in Report of Bristol Conference on the Strength of Solids (The Physical Society, London, 1948 ).

2) J. W. Christian, Proc. Roy. Soc. Lond. A 206, 51 (1951).

3) T. Ericsson, Acta. Met. 14, 853 (1966).

4) G. B. O1son and M. Cohen, Met. Trans. A I, 1897 (1976).

5) J. W. Christian, The Theory of Transformations in Metals and Alloys (Pergamon, New York, 1965).

6) J. P. Hirth and J. Lothe, Theory of Dislocations, (McGraw-Hil1, New York, 1968).

7) H. Kronmuller, Phys, stat. So1. B 52, 231 (1972).

8) W. F. Weston and A. V. Granato, Phys. Rev. B 12, 5355 (1975). 